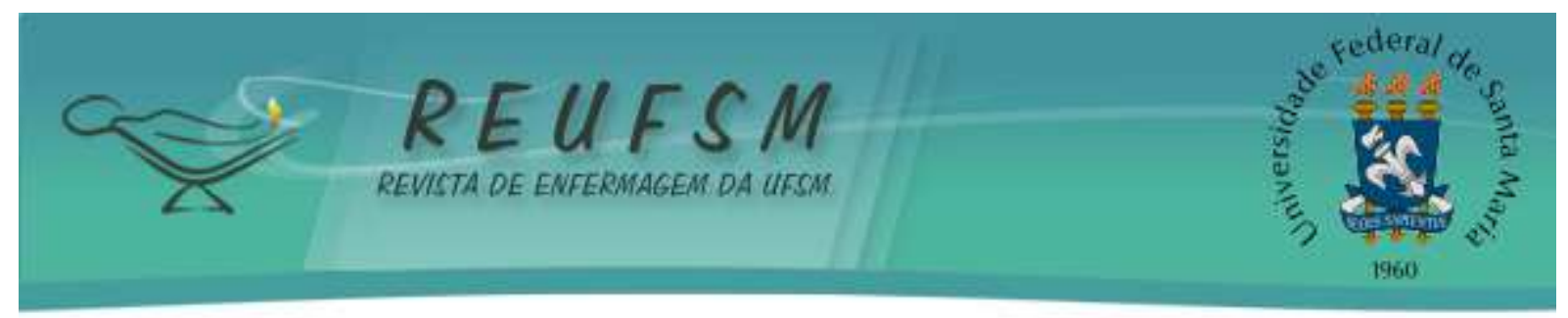

ARTIGO ORIGINAL

\title{
ACOMPANHAMENTO DE CRIANÇAS EXPOSTAS AO HIV MATERNO NO MUNICÍPIO DE CANOAS/RS
}

\author{
THE MONITORING OF CHILDREN EXPOSED TO MATERNAL HIV IN THE \\ MUNICIPALITY OF CANOAS / RS
}

\section{ACOMPAÑAMIENTO DE NIÑOS EXPUESTOS AL VIH MATERNO EN EL MUNICIPIO DE CANOAS/RS}

\author{
Maria Renita Burg ${ }^{1}$ \\ Márcia Andreazza ${ }^{2}$ \\ Sinara Rocha e Silva ${ }^{3}$ \\ Maria Marilene Trindade de Oliveira ${ }^{4}$ \\ Simone dos Santos 5 \\ Neiva Isabel Raffo ${ }^{6}$
}

\section{Doi: $10.5902 / 2179769223608$}

RESUMO: Objetivos: estimar a taxa de transmissão vertical do HIV em crianças residentes de Canoas/RS entre 2012 e 2013; e investigar dados sociodemográficos e de cuidados das crianças e mães no mesmo período. Método: estudo de coorte prospectivo, formado por todos os 45 recém-nascidos vivos e expostos ao HIV, nascidos no período de junho de 2012 a dezembro de 2013, cujas mães foram acompanhadas pelo Serviço de Atendimento Especializado (SAE) de Canoas/RS, até a definição da sorologia para o HIV na criança. O cálculo da taxa de transmissão do HIV foi realizado com 41 crianças que tiveram efetivamente o resultado da exposição ao HIV materno. Resultados: a taxa de transmissão vertical do HIV foi de 4,88\%, atribuída à baixa adesão de consultas de pré-natal, à interrupção do antirretroviral profilático durante a gestação e ao uso de drogas ilícitas pelas mulheres. Conclusão: as informações advindas deste estudo permitirão aos gestores do município planejar intervenções que possam contribuir na erradicação da transmissão perinatal do HIV como forma de contágio.

Descritores: Sorodiagnóstico da AIDS; Transmissão vertical de doença infecciosa; Recém-nascido.

ABSTRACT: Aim: to estimate the vertical HIV transmission rate in children living in Canoas / RS between 2012 and 2013; and to investigate sociodemographic and care data of children and mothers in the same time period. Method: prospective cohort study, comprised by 45 living

\footnotetext{
${ }^{1}$ Enfermeira. Mestre em Saúde Coletiva. Professora da Universidade Luterana do Brasil-ULBRA. Canoas, RSBrasil. Coordenadora da Residência Multiprofissional em Saúde Comunitária. Email: renitaburgfigueredo@gmail.com

${ }^{2}$ Enfermeira da Unimed de Porto Alegre. Egressa do Curso de Graduação de Enfermagem da Ulbra/Canoas. Email: marcia.andreazza@unimedpoa.com.br

${ }^{3}$ Enfermeira de UPA 24 horas do município de Canoas, RS-Brasil. Egressa do Curso de Graduação de Enfermagem da Ulbra/Canoas. Email: rocha.sinara@yahoo.com.br

${ }^{4}$ Enfermeira. Egressa do Curso de Enfermagem da Universidade Luterana do Brasil-ULBRA. Canoas, RSBrasil. Email: Iamnursetwo@ hotmail.com

${ }^{5}$ Enfermeira. Especialista na Atenção Básica/Saúde da Família pela Residência Multiprofissional na Saúde. Canoas, RS-Brasil Email: santos.simonefisio@yahoo.com.br

${ }^{6}$ Enfermeira. Mestre em Epidemiologia. Servidora da Secretaria Municipal da Saúde de Porto Alegre. Email: neivarw@gmail.com
} 


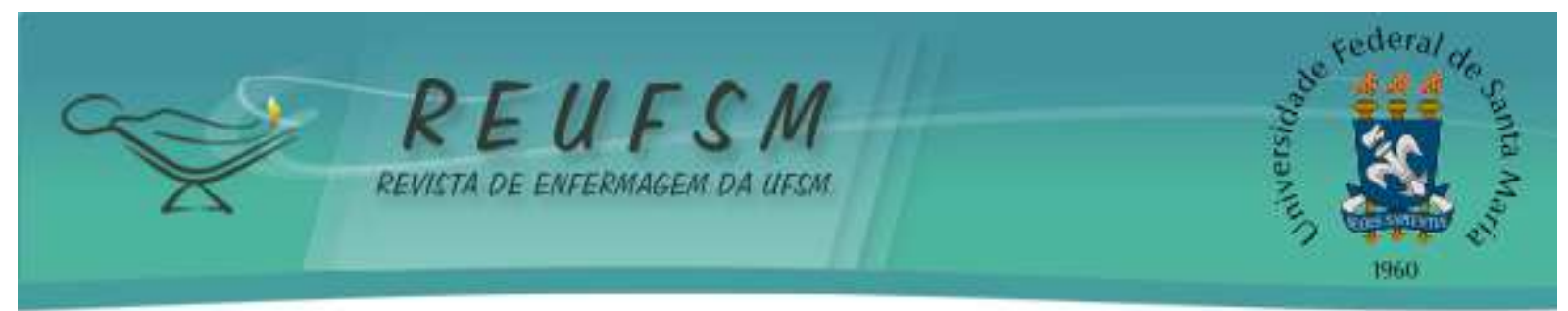

newborns who were exposed to HIV, all born between June 2012 and December 2013, whose mothers were accompanied by Service Specialized-SAE Canoas / RS, to the definition of serology for HIV in children. The calculation of HIV transmission rates was accomplished with 41 children who effectively had the result of exposure to maternal HIV. Results: The vertical HIV transmission rate was $4.88 \%$, attributed to the low adherence to prenatal consultations, to a discontinuation of prophylactic ARV during gestation and to the use of illicit drugs by women. Conclusion: The information from this study will enable municipal managers to plan interventions that may contribute to the eradication of perinatal transmission of HIV as a form of contagion.

Descriptors: AIDS serodiagnosis; Infectious disease transmission, vertical; Infant, Newborn.

RESUMEN: Objetivo: estimar la tasa de transmisión vertical del VIH en niños residentes de Canoas/RS, entre 2012 y 2013; e investigar datos sociodemográficos de cuidado de los niños y madres en el mismo período. Método: estudio de cohorte prospectivo, formado por todos los 45 recién nacidos vivos y expuestos al VIH, nacidos en el período de junio de 2012 a diciembre de 2013, cuyas madres fueron acompañadas por el Servicio de Atención Especializada-SAE de Canoas/RS, hasta La definición de la serología para el VIH en el niño. El cálculo de la tasa de transmisión del VIH fue realizado con 41 niños, que tuvieron efectivamente el resultado de la exposición al VIH materno. Resultados: La tasa de transmisión vertical del VIH encontrada fue de 4,88\%, atribuida a la baja adhesión a las consultas prenatal, a la interrupción del ARV profiláctico durante la gestación y al uso de drogas ilícitas por las mujeres. Conclusión: Las informaciones provenientes de este estudio permitirán a los gestores del municipio planificar intervenciones que puedan contribuir con la erradicación de la transmisión perinatal del VIH como forma de contagio.

Descriptores: Serodiagnóstico del SIDA; Transmisión vertical de enfermedad infecciosa; Recién nacido.

\section{INTRODUÇÃO}

A infecção por HIV segue como um importante problema de saúde no ranking brasileiro e mundial, sendo que a propagação em todos os continentes está associada às desigualdades sociais e de gênero. ${ }^{1}$

No Brasil, o aumento da epidemia de HIV/Aids, na população heterossexual, está diretamente relacionada à incidência de aids em mulheres. Esta situação tem acarretado um incremento no número de crianças infectadas pelo HIV. A transmissão vertical (TV) é responsável por $84 \%$ dos casos de Aids em crianças. ${ }^{2}$

Já a prevalência da infecção por HIV em grávidas está em $0,38 \%$ e tal aumento de gestantes infectadas exigiu ações por parte do Ministério da Saúde. Assim, o ano 2000 iniciou com a obrigatoriedade da notificação compulsória de gestantes e crianças expostas ao HIV. ${ }^{3}$

A transmissão vertical do HIV pode ocorrer de diversas formas: $35 \%$ durante a gestação; $65 \%$ no trabalho de parto ou no parto e $7 \%$ a $22 \%$ a cada mamada. O Ministério da Saúde, através do Programa Nacional de DST/Aids, estima a transmissão em cerca de $26 \%$ 


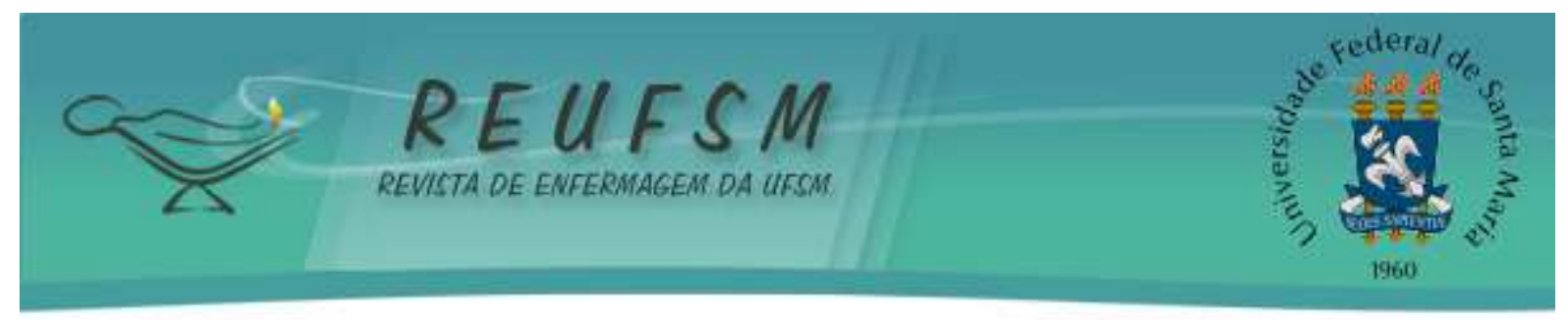

das gestações, caso não ocorra nenhuma intervenção de profilaxia, mas pode ser reduzida para menos de $2 \%$ com intervenções preconizadas. ${ }^{4}$

O primeiro país a receber a validação da OMS em relação à eliminação da TV do HIV foi Cuba, em 2015. Apresentou taxas de transmissão inferior a 5\% em recém-nascidos amamentados ao seio, e inferior a $2 \%$ naqueles que receberam a fórmula láctea para alimentação. O uso de antirretrovirais (ARV) das gestantes foi de $95 \% .^{3}$

$\mathrm{Na}$ primeira consulta de pré-natal, a gestante tem a indicação de realizar os testes rápidos para detectar a hepatite $\mathrm{B}$ e $\mathrm{C}$, sífilis e anti-HIV, importantes para evitar a transmissão destas doenças da mãe para o filho. Caso a gestante não realize consulta de pré-natal, os testes rápidos podem ser realizados na maternidade, no momento do parto. Se o teste de HIV for positivo no pré-natal, é indicado o tratamento com os medicamentos ARV para prevenir a transmissão ao feto. O referido tratamento ocorre também no parto. Da mesma forma, o recém-nascido deve fazer uso do medicamento ARV xarope por quatro semanas. ${ }^{5}$

O pré-natal da gestante soropositiva se diferencia em alguns aspectos em relação ao pré-natal das demais mulheres que não apresentam a soropositividade, principalmente no que diz respeito à saúde do bebê destas mães. É necessário um cuidado contínuo durante toda a gestação com a realização de exames específicos (CD4 e carga viral), tratamento medicamentoso e amparo psicológico. ${ }^{3,5}$

Como a transmissão do HIV também pode acontecer durante a amamentação, por meio do leite materno, a mãe que tem o vírus não deve amamentar o bebê, sob o risco de transmissão da doença para o seu filho. É orientada a suspensão da amamentação e a inibição da lactação. Portanto, o leite da mãe deve ser substituído por leite artificial. ${ }^{5}$

No Brasil, de acordo com o Sistema de Informações de Agravos de Notificação- SINAN, entre 2000 até junho de 2015, foram notificadas 92.210 gestantes infectadas com o HIV, a maioria destas residentes na região Sudeste (40,5\%), seguida pelas regiões Sul (30,8\%). Já, em 2014, foram identificadas 7.668 gestantes, sendo a região sul $(28,1 \%)$ a região que apresentou a maior taxa de detecção entre as regiões, aproximadamente 2,1 vezes maior que a taxa do Brasil. O Rio Grande do Sul tem 8,8 casos para cada mil nascidos vivos. ${ }^{6}$ Canoas é a quarta cidade do Estado no ranking de HIV, com taxa de incidência de 58,5 casos por 100 mil habitantes. ${ }^{6}$ 


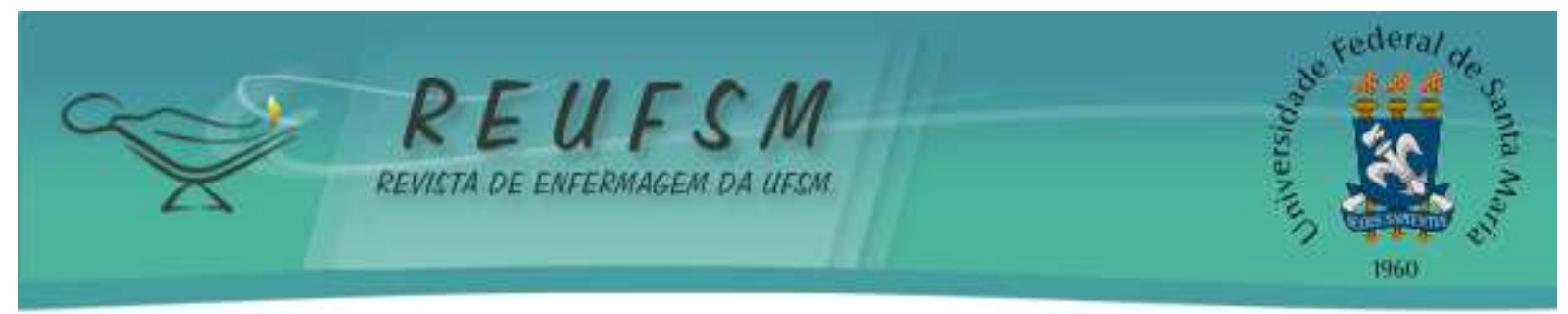

O município em estudo não apresenta nenhuma pesquisa avaliando a taxa de transmissão vertical pelo HIV, sendo assim é difícil identificar a realidade das gestantes soropositivas e avaliar a qualidade do pré-natal oferecido.

Os resultados desta pesquisa possibilitam ao município conhecer este indicador, contribuindo com a avaliação da qualidade da assistência pré-natal das gestantes soropositivas para o HIV. Além disto, este estudo acompanhou regularmente as crianças expostas desde o nascimento até o diagnóstico da sorologia para o HIV.

Neste contexto, esta pesquisa tem por objetivos estimar a taxa de transmissão vertical do HIV em crianças residentes de Canoas/RS entre 2012 e 2013; e investigar dados sociodemográficos e de cuidados das crianças e mães no mesmo período.

\section{MÉTODO}

Trata-se de estudo observacional, de coorte prospectivo, que propõe estimar a taxa de transmissão vertical, em Canoas/RS, e descrever as características relacionadas ao pré-natal, parto e realização de medidas de profilaxia da infecção pelo HIV por transmissão vertical.

O Município de Canoas está localizado na região metropolitana de Porto Alegre, sendo o município do Estado do Rio Grande do Sul mais populoso da região metropolitana. ${ }^{7}$

O Serviço de Atendimento Especializado (SAE) de Canoas/RS iniciou suas atividades em dezembro de 1997 e atende 2.555 pacientes infectados pelo vírus HIV que seguem acompanhamento regular no serviço. Dentre eles, 1.345 fazem uso de medicações antirretrovirais, sendo acompanhadas, em média, 50 gestantes. Este serviço é a única referência no município para acompanhar gestantes soropositivas e crianças expostas ao HIV. Ainda que o pré-natal não tenha sido realizado no SAE, no momento do parto a criança exposta ao HIV era inserida neste estudo, quando na maternidade e, posteriormente, acompanhada no SAE pelo serviço ou por busca ativa pela equipe de pesquisa.

Foram incluídos no estudo 45 recém-nascidos vivos, expostos ao HIV materno, nascidos no período de junho de 2012 a dezembro de 2013, e que ingressaram no SAE de Canoas para acompanhamento pediátrico. O critério para entrada do recém-nascido no estudo foi ser filho de gestantes soropositivas, com pré-natal realizado em Canoas e acompanhadas no SAE. 


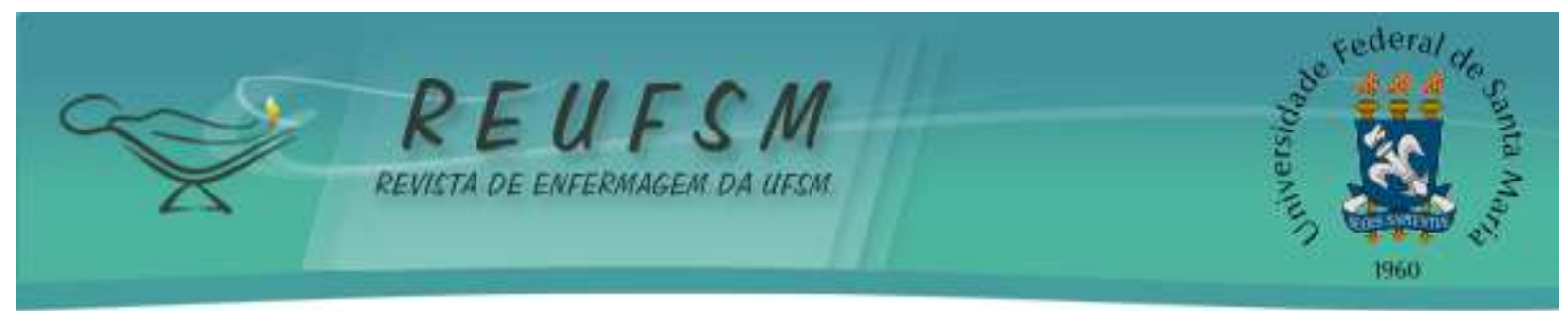

Todas as crianças expostas ao HIV neste período, no município, foram acompanhadas no SAE, de acordo com os gestores do serviço, que alimentam os dados no SINAN de gestante HIV e criança exposta.

A captação dos recém-nascidos para o estudo ocorreu quando a criança era inserida no Projeto Nascer (projeto realizado em parceria com Ministério da Saúde, em que as mães ou responsáveis se inscreviam para o recebimento mensal das latas com a fórmula láctea até os 18 meses de vida), ou eram trazidas para o primeiro atendimento pediátrico no SAE. A mãe da criança exposta ao HIV (ou responsável), após a concordância em participar da pesquisa, assinou o Termo de Consentimento Livre e Esclarecido. Para as mães que não compareceram regularmente nas consultas de acompanhamento com seus filhos, no SAE, os pesquisadores fizeram contato telefônico com as mesmas, solicitando a presença dos mesmos no serviço.

As variáveis usadas para investigar as gestantes foram: idade; raça; escolaridade; uso de drogas; momento do diagnóstico HIV; uso do ARV; parceiro com HIV e ou Aids; uso de drogas. As variáveis relativas às crianças expostas: sexo; resultado VDRL; aleitamento materno; inicio tomada ARV e o tempo que fez uso.

A presente pesquisa foi aprovada pelo Comitê de Ética, em pesquisa da Universidade Luterana do Brasil, sob o Parecer Consubstanciado no 23536213.8.0000.5349.

As entrevistas foram realizadas no serviço, em sala reservada, momento em que foram coletados os dados relacionados ao pré-natal e parto. Além da entrevista com o preenchimento do instrumento de coleta de dados, também foram buscados dados secundários em prontuários, das gestantes, das mães, e crianças, arquivados no SAE e no Hospital Universitário, maternidade de referência no município.

Os dados foram armazenados no programa Microsoft Office Excel® (2007) e analisados através de software SPSS versão13.0. As variáveis em estudo foram apresentadas por meio das frequências absolutas e relativas e estão apresentadas nas tabelas: 1; 2 e 3 e no gráfico 1 .

\section{RESULTADOS}

Entre as 45 crianças incluídas no estudo, foram acompanhadas até o desfecho da exposição ao HIV 41 crianças $(91,11 \%)$. As perdas do estudo consistem em quatro casos, cujo desfecho da exposição não foi conhecido $(8,89 \%)$. Na análise dos resultados, foram mantidos os dados dos 45 sujeitos que entraram no estudo, mesmo aqueles cujos efeitos da exposição 


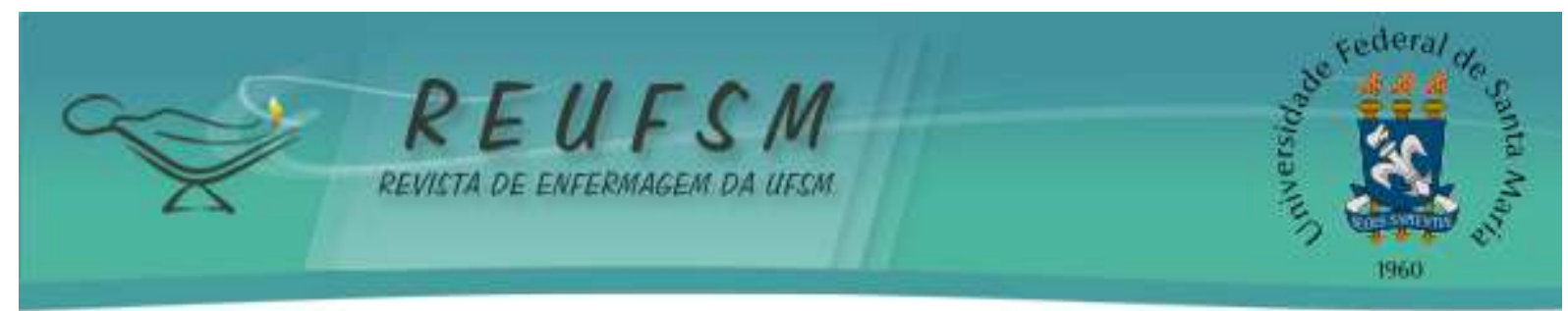

ao HIV materno não foram conhecidos até o término da pesquisa. Entretanto, no cálculo de transmissão da taxa do HIV, foram consideradas apenas as 41 crianças que tiveram efetivamente o resultado da exposição ao HIV materno.

O acompanhamento não foi concluído em 4 casos: um caso (2,2\%), cujo motivo foi o óbito da criança; em dois casos (4,4\%), houve abandono do acompanhamento no SAE/Canoas pela família; e para um caso(2,2\%), a família mudou-se de município e de residência, não localizada pelos pesquisadores.

Com relação aos dois casos de abandono do acompanhamento, um deles o prontuário da criança estava sem informações, e o prontuário da mãe não foi localizado no SAE. Neste caso, o Conselho Tutelar da região realizou diversas buscas ativas, para localizar a família na cidade, sem sucesso até o término da pesquisa. Quanto à outra criança, a mãe realizou somente uma consulta de pré-natal, não coletou teste rápido no parto. A mãe não queria que o restante da família soubesse de sua situação sorológica para o HIV. O caso foi encaminhado para o Conselho Tutelar, mas sem resultado até o término da pesquisa, relacionado à localização da família.

A Tabela 1 mostra perfil sociodemográfico e dados de saúde das 45 mães das crianças em estudo.

Tabela 1- Perfil sociodemográfico e dados de saúde das mães das crianças do estudo. Canoas/RS, 2015.

\begin{tabular}{|c|c|c|}
\hline Variável & $\mathbf{n}$ & $\%$ \\
\hline \multicolumn{3}{|l|}{ Idade } \\
\hline$<18$ anos & 4 & 8,9 \\
\hline $18-24$ anos & 14 & 31,1 \\
\hline 25-30 anos & 12 & 26,7 \\
\hline$>30$ anos & 15 & 33,3 \\
\hline \multicolumn{3}{|l|}{ Raça } \\
\hline Branca & 27 & 60 \\
\hline Preta & 6 & 13,3 \\
\hline Parda & 9 & 20 \\
\hline Sem registro & 3 & 6,7 \\
\hline \multicolumn{3}{|l|}{ Escolaridade } \\
\hline Nenhuma & 8 & 17,8 \\
\hline Ensino Fundamental incompleto & 24 & 53,3 \\
\hline Ensino fundamental Completo & 11 & 24,4 \\
\hline Superior Incompleto & 2 & 4,4 \\
\hline \multicolumn{3}{|l|}{ Uso de drogas } \\
\hline Sim & 13 & 28,9 \\
\hline Não & 25 & 55,6 \\
\hline Sem registro & 7 & 15,6 \\
\hline
\end{tabular}




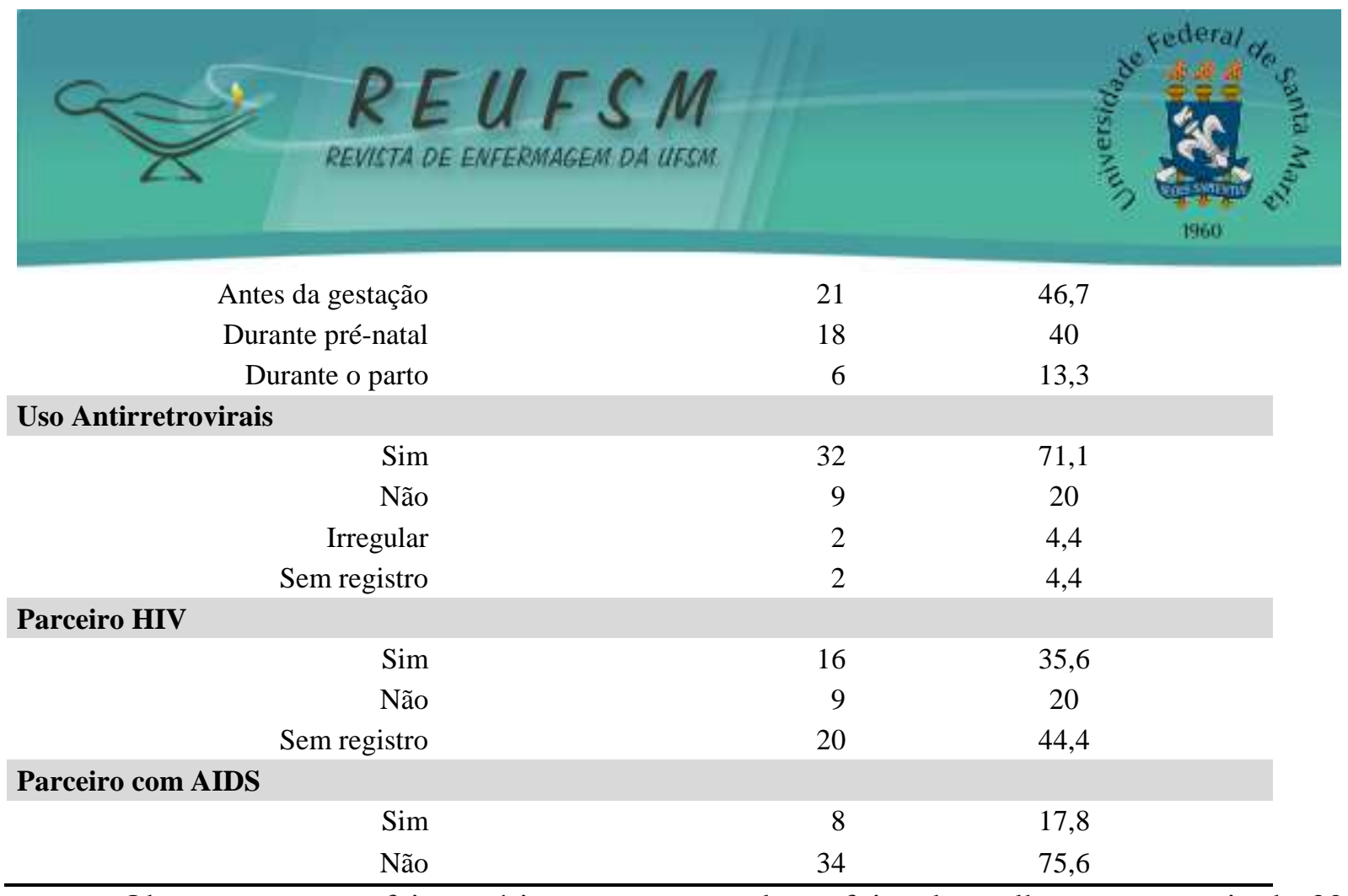

Observa-se que a faixa etária materna prevalente foi a de mulheres com mais de 30 anos, com mediana de 28 anos. A raça branca representou $60 \%$ da população estudada e a raça preta e parda somadas, perfizeram 33,30\%. Quanto à escolaridade, observou-se que $17,80 \%$ das gestantes não tinha nenhum ano de estudo; 53,3\% tinham ensino fundamental incompleto; $24,4 \%$ ensino fundamental completo.

O bairro do município com a maior incidência de gestantes (40\%) foi o Mathias Velho. Este bairro possui altos índices de violência e é o mais populoso do município com 48.806 habitantes conforme censo do IBGE em $2010 .^{7}$ A população do território é atendida por equipes de Estratégia de Saúde da Família em seis Unidades Básicas de Saúde.

Em relação à detecção do HIV, 46,7\% já tinham diagnóstico da infecção ao engravidarem; $40 \%$ ficaram sabendo da infecção durante o pré-natal; $13,3 \%$ no momento do parto. Faziam uso de ARV regularmente $71,1 \%$ das gestantes e $24,4 \%$ não faziam uso ou o uso era irregular.

Em relação aos dados do parceiro atual (no momento do estudo) das gestantes, 35,60\% tinham conhecimento de resultado de exame positivo para o HIV e 17,80\% já tinham diagnóstico de AIDS.

No Gráfico 1, verificam-se as drogas mais utilizadas pelas mães durante a gestação. Entre as drogas consideradas lícitas: 33,3\% das gestantes admitiram o uso do fumo e 8,9\%, de álcool; entre as ilícitas: 13,3\% referiram uso de crack, 4,4\% de maconha, 4,4\% de cocaína e $2,2 \%$ de inalantes como a cola.

Gráfico 1- Tipo de drogas mais utilizadas pelas mães durante a gestação. Canoas/RS,2015. 

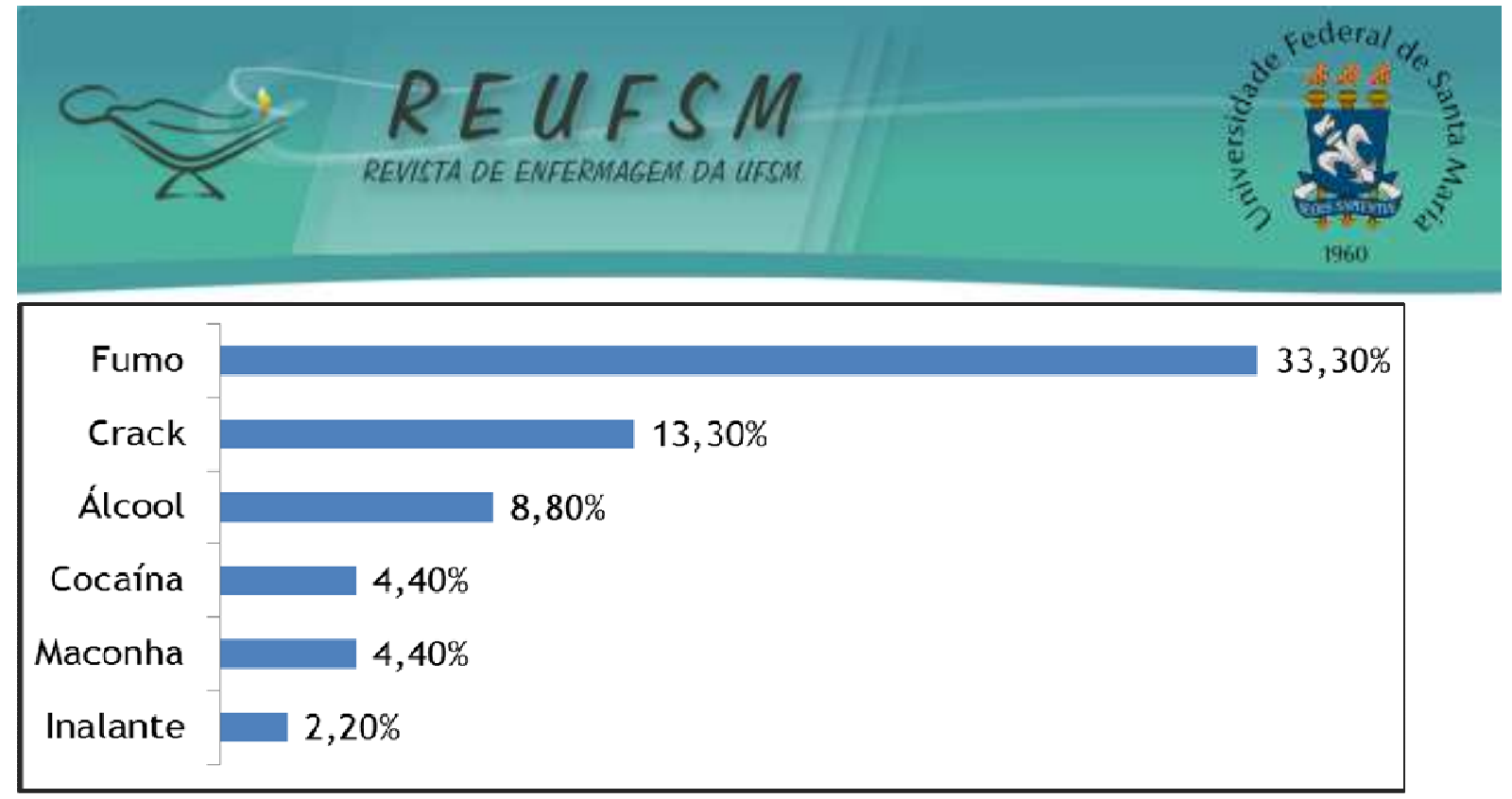

A Tabela 2 informa as características relacionadas ao pré-natal e parto das mães das crianças do estudo, o que mostra que $15,5 \%$ das gestantes não realizaram pré-natal; 35,6\% realizaram menos de 6 consultas. Não se obteve esta informação para 11,1\%. O parto ocorreu para 75,6\% das gestantes no Hospital Universitário, único hospital com maternidade no município. Ainda em 24,4\%, o parto deu-se em algum hospital da capital (Porto Alegre).

Tabela 2 - Características relacionadas ao pré-natal e parto das mães das crianças do estudo. Canoas/RS, 2015.

\begin{tabular}{|c|c|c|}
\hline Variável & $\mathbf{n}$ & $\%$ \\
\hline \multicolumn{3}{|l|}{$\mathrm{N}^{0}$ consultas pré-natal } \\
\hline Sem consulta & 7 & 15,5 \\
\hline Menos de 6 & 16 & 35,6 \\
\hline 6 a 9 & 17 & 37,8 \\
\hline Sem registro & 5 & 11,1 \\
\hline \multicolumn{3}{|l|}{ Local do parto } \\
\hline Hospital Universitário/Canoas & 34 & 75,6 \\
\hline Hospitais de Porto Alegre & 11 & 24,4 \\
\hline \multicolumn{3}{|l|}{ Tipo de parto } \\
\hline Normal & 13 & 28,9 \\
\hline Cesárea & 30 & 66,7 \\
\hline Sem registro & 2 & 4,4 \\
\hline \multicolumn{3}{|l|}{ Uso ARV parto } \\
\hline Sim & 40 & 88,9 \\
\hline Não & 5 & 11,1 \\
\hline
\end{tabular}




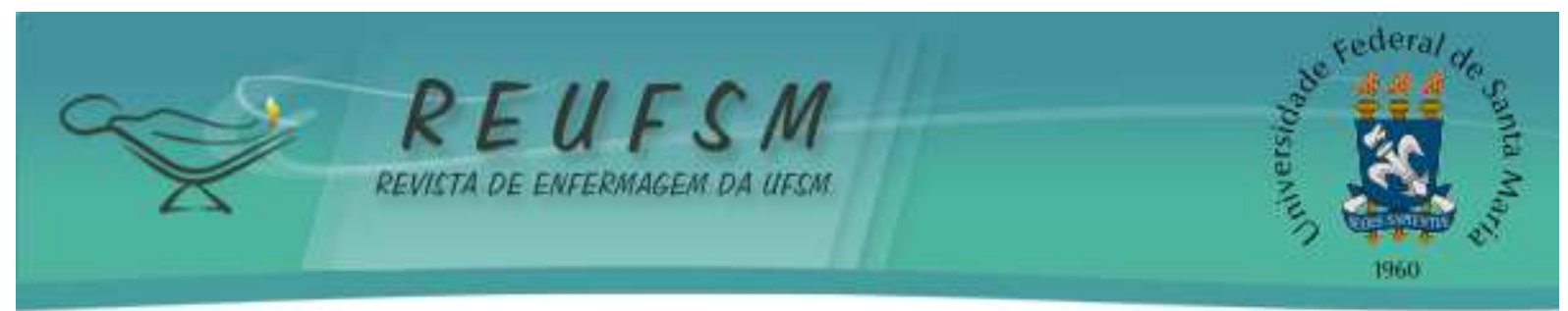

O parto cesáreo ocorreu em $66,7 \%$ dos casos, e o parto normal em $28,9 \%$, sendo que $11,1 \%$ não fizeram uso de ARV durante o parto.

A Tabela 3 apresenta as características relacionadas às crianças expostas ao HIV no período perinatal, nascidos no período de junho de 2012 a dezembro de 2013 e que ingressaram no SAE/Canoas para acompanhamento. Observamos que 51,1\% das crianças eram do sexo feminino e $48,9 \%$ do masculino.

O teste de sífilis mostrou-se reagente para 4,4\% dos recém-nascidos, sendo que para $62,2 \%$ destas crianças o exame não foi realizado. Observou-se que 97,8\% das crianças não receberam aleitamento materno, sendo que 97,8\% dos recém-nascidos receberam ARV profilático nas primeiras 24 horas de vida, e 95,6\% destes seguiram o uso por 6 semanas, segundo protocolo do Ministério da Saúde naquele período.

Tabela 3 - Características relacionadas às crianças expostas ao HIV no período perinatal, nascidos no período de junho de 2012 a dezembro de 2013. Canoas/RS, 2015.

\begin{tabular}{|c|c|c|}
\hline Variável & $\mathbf{n}$ & $\%$ \\
\hline \multicolumn{3}{|l|}{ Sexo } \\
\hline Feminino & 23 & 51,1 \\
\hline Masculino & 22 & 48,9 \\
\hline \multicolumn{3}{|l|}{ Resultado do VDRL na criança } \\
\hline Não reagente & 15 & 33,3 \\
\hline Reagente & 2 & 4,4 \\
\hline Não fez o exame & 28 & 62,2 \\
\hline \multicolumn{3}{|l|}{ Aleitamento materno } \\
\hline Sim & 1 & 2,2 \\
\hline Não & 44 & 97,8 \\
\hline \multicolumn{3}{|l|}{ Início do ARV pelo recém-nascido } \\
\hline Primeiras 24 horas & 44 & 97,8 \\
\hline Não fez & 1 & 2,2 \\
\hline \multicolumn{3}{|l|}{ Tempo ARV pela criança } \\
\hline 6 semanas & 43 & 95,6 \\
\hline Só no hospital & 1 & 2,2 \\
\hline Não tomou & 1 & 2,2 \\
\hline
\end{tabular}

Das 41 crianças que permaneceram no estudo até o diagnóstico da exposição ao HIV materno, $95,12 \%$ (39 crianças) foram consideradas negativas e 4,88\% (duas crianças) foram consideradas infectadas pelo HIV. 


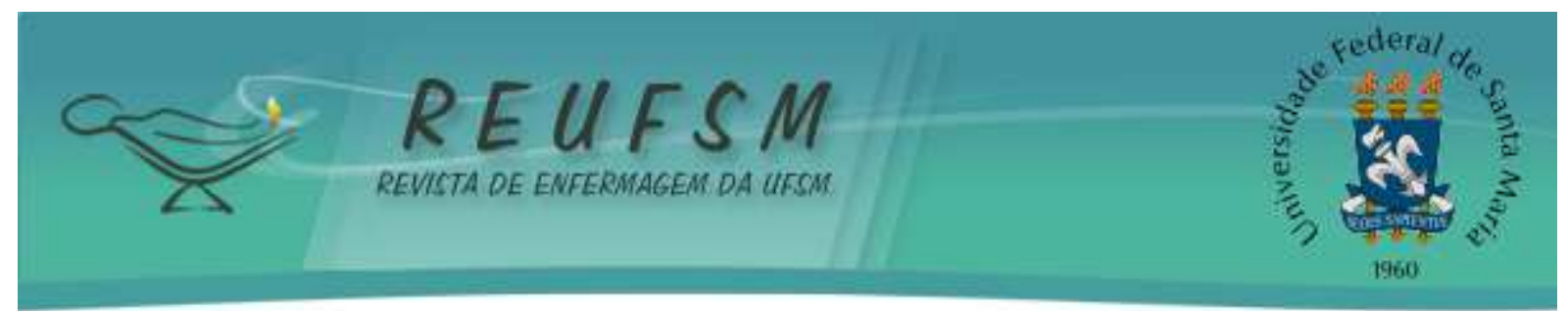

Nos dois casos em que ocorreu a transmissão vertical do HIV, verificou-se uma baixa adesão ao pré-natal, sendo que uma gestante realizou apenas uma consulta e a outra, apenas duas consultas. $\mathrm{O}$ uso de drogas ilícitas foi detectado em ambas as mães que também eram tabagistas. $\mathrm{O}$ tratamento profilático foi interrompido por estas mulheres durante a gestação. Entretanto, em ambos os casos foram utilizados ARV, durante o parto, e foi administrado o xarope na criança, nas primeiras 24 horas no ambiente hospitalar. As mães referiram que realizaram durante seis semanas o tratamento com o xarope. Também referiram que nenhuma das duas crianças recebeu aleitamento materno e ambas receberam a fórmula láctea para alimentação.

\section{DISCUSSÃO}

As gestantes/mães das crianças do estudo, em sua maioria, apresentaram idade igual ou superior a 25 anos - (60,0\%). Este dado vem ao encontro de pesquisa com gestantes HIV, sendo que $48,3 \%$ tinham entre 25 a 34 anos. $^{8}$ Esta faixa etária também está em acordo com os dados nacionais que apontam que a maior concentração de caso de aids, no Brasil, está nos indivíduos com idade de 25 e 39 anos, em ambos os sexo. ${ }^{4}$

A raça predominante foi a branca em $60 \%$, mas a raça preto-parda esteve presente em um terço das gestantes/mães. Resultados semelhantes foram encontrados em outros estudos, ${ }^{9-10}$ em que a distribuição racial foi de $57 \%$ de gestantes brancas e $23 \%$ pardas ou mestiças. Na pesquisa conduzida pelo Ministério da Saúde, 44,8\% se auto definiram brancas e 40,6\% pardas. ${ }^{6}$

Em relação à escolaridade, pode-se inferir que era baixa entre estas mulheres, pois $71,1 \%$ não estudaram ou não chegaram a concluir o ensino fundamental. Apenas 4,4\% destas gestantes/mães tiveram acesso ao ensino superior. Em estudos realizados em outras capitais, verificou-se também uma baixa escolaridade: em Espírito Santo, 47,6\% das gestantes tinham apenas de 4 a 7 anos de escolaridade e, em Belo Horizonte, apenas um terço das gestantes tinham de 8 a 12 anos de escolaridade. 8,10 $^{-10}$

As gestantes deste estudo são oriundas de bairros com grande vulnerabilidade social. As condições socioeconômicas desfavoráveis levam à vulnerabilidade de bairros do município e podem estar afetando diretamente a vida destas mulheres e seus descendentes, bem como refletindo na qualidade de vida das mesmas. ${ }^{11-12}$

Neste estudo encontramos $28,9 \%$ de gestantes fazendo uso de drogas, expondo a saúde dos filhos que estão gerando. As drogas lícitas mais utilizadas foram o fumo e o álcool. Um terço das gestantes era fumante e cerca de 9\% usava álcool. Entre as drogas ilícitas, verificou-se o uso de crack, 


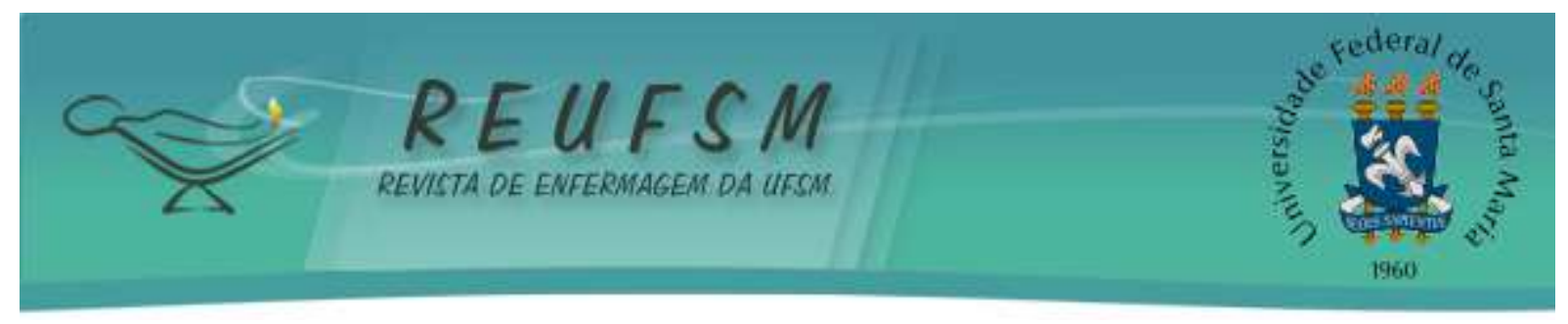

maconha e cocaína. Em outro estudo realizado com esta mesma população, verificou-se que algumas mães, ao longo de suas histórias, se desestruturam, buscando refúgio nas drogas ilícitas e até mesmo nas lícitas, quando estas últimas são utilizadas de forma indiscriminada. Ficou evidenciado nos depoimentos, que o uso de drogas foi motivado pela descoberta do diagnóstico da infecção pelo HIV, e, ainda, pela possibilidade de transmissão do vírus ao filho que está gerando. ${ }^{13}$

O uso de drogas na gravidez acarreta riscos únicos como problemas de saúde física, desnutrição e susceptibilidade a infecções, os quais podem ser transmitidos ao feto em desenvolvimento. ${ }^{14}$ Em um estudo sobre o perfil epidemiológico das gestantes infectadas pelo HIV, realizado em Santa Maria/RS, observou-se 10,1\% das gestantes utilizaram drogas, índice bem menor daquele encontrado no presente estudo. ${ }^{9}$ Ainda em relação ao pré-natal, chama a atenção para o fato de que 15,5\% não fizeram consultas de pré-natal.

A adesão das gestantes ao pré-natal foi baixa, visto que 15,5\% não realizaram pré-natal, o que põe em risco a saúde do binômio mãe-filho, além da perda de oportunidades, tanto para o diagnóstico precoce como para o início da profilaxia antirretroviral. Entre as gestantes que realizaram pré-natal, cerca de um terço realizou menos de 6 consultas. O Ministério da Saúde, em 2000, lançou o Programa de Humanização do Pré-Natal e Nascimento (PHPN), incentivando as gestantes a buscarem o Sistema Único de Saúde e estabelecendo que sejam realizadas, no mínimo, seis consultas de pré-natal. Também seguem o preconizado no Plano Municipal da Saúde do município de Canoas, que tem como meta sete consultas de pré-natal. ${ }^{6,15}$

Mais da metade das gestantes realizaram parto por cesariana e cerca de $11 \%$ não receberam a antirretroviral no parto, evidenciando falhas na captação destas gestantes precocemente e na profilaxia da transmissão vertical do HIV. Os resultados de um estudo realizado no Ceará ${ }^{16}$ mostra que $100 \%$ das gestantes realizaram o pré-natal, mas também $50 \%$ realizou de 4 a 6 consultas, o número menor de consultas preconizado pelo Ministério da Saúde. Naquele estudo, o parto mais utilizado também foi a cesárea, com $72,7 \%$ e foi administrado antirretroviral intraparto em $77,3 \%$ das parturientes. ${ }^{16}$ Em outra pesquisa, $51,7 \%$ das gestantes aderiam à medicação, e estas gestantes, que eram mais escolarizadas, começaram o pré-natal antes, realizaram mais consultas e referiram maior apoio emocional. ${ }^{17}$

O Ministério da Saúde indica para a gestante soropositiva para o HIV, a infusão endovenosa de Zidovudina (AZT), no início do trabalho de parto, até o nascimento do bebê, 


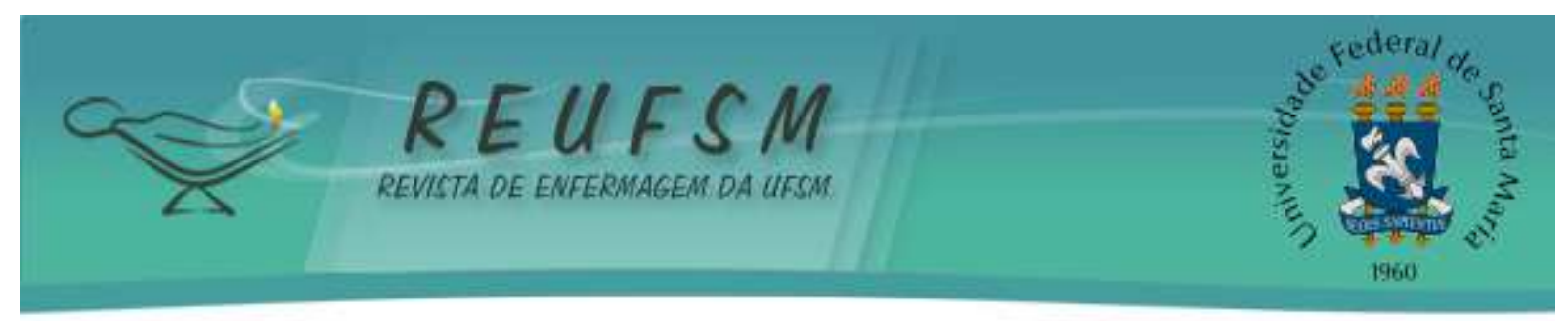

independentemente do esquema ARV utilizado no pré-natal. Em relação à via de parto, a cesárea eletiva reduz o risco de transmissão vertical. ${ }^{3,5}$

Neste estudo, 97,8\% das crianças receberam ARV nas primeiras 24 horas de vida e 2,2\% não fizeram uso. O Ministério da Saúde recomenda que, para estes recém-nascidos, o leite materno seja substituído pela fórmula láctea dispensada gratuitamente nos serviços que acompanham estas crianças. ${ }^{3,5}$ Entretanto, o aleitamento materno ocorreu em 2,2\% destas crianças.

Para 95,6\% das crianças, receberam ARV conforme recomendado, sendo que 2,2\% só utilizaram o medicamento durante a hospitalização e 2,2\% não fizeram uso. Atualmente, é preconizado pelo Ministério da Saúde o uso de AZT xarope para o recém-nascido, iniciando nas duas primeiras horas de vida, concluindo em quatro semanas, pelo Ministério da Saúde. ${ }^{5}$ Em estudo realizado, no Ceará, $91 \%$ das crianças receberam ARV nas primeiras 24 horas de vida. ${ }^{16}$

No estudo de Alagoas, apenas 14,3\% das crianças realizaram coleta da terceira carga viral. ${ }^{11}$ Na maternidade de referência de Sergipe, foram avaliadas 110 gestantes e os recémnascidos, sendo que duas crianças $(1,8 \%)$ foram consideradas infectadas, e 50 (45,5\%) não tinham o diagnóstico definido. ${ }^{17}$

Em estudo transversal, realizado no SAE, em Alagoas, foram investigadas 76 crianças expostas por transmissão vertical do HIV das quais cinco foram infectadas. A prevalência foi de $6,6 \%$ de transmissão vertical. ${ }^{11}$

Nesta pesquisa, a taxa de transmissão vertical encontrada foi de 4,88\%. A mesma é superior às taxas de transmissão vertical dos países desenvolvidos que é inferior a 1\%. O indicador brasileiro é de $2,7 \%$, bem como o do Rio Grande do Sul, em 2011, foi de 3,3\%. ${ }^{3}$

\section{CONCLUSÃO}

As limitações deste estudo foram em relação aos dados coletados nos prontuários das gestantes no Hospital Universitário e no SAE. Os registros nem sempre eram adequados e suficientes e podem ter comprometido a qualidade dos dados obtidos. Mesmo após a revisão de outras fontes de dados, buscando complementação das variáveis pesquisadas, faltaram informações, o que limitou a análise de algumas variáveis como a carga viral da gestante; o uso de drogas; uso de ARV; se o parceiro era soropositivo para HIV, favorecendo perdas. Também a efetiva presença da mãe no SAE com seu filho para acompanhamento e complemento da coleta de dados, se tornou possível após várias consultas desmarcadas. 


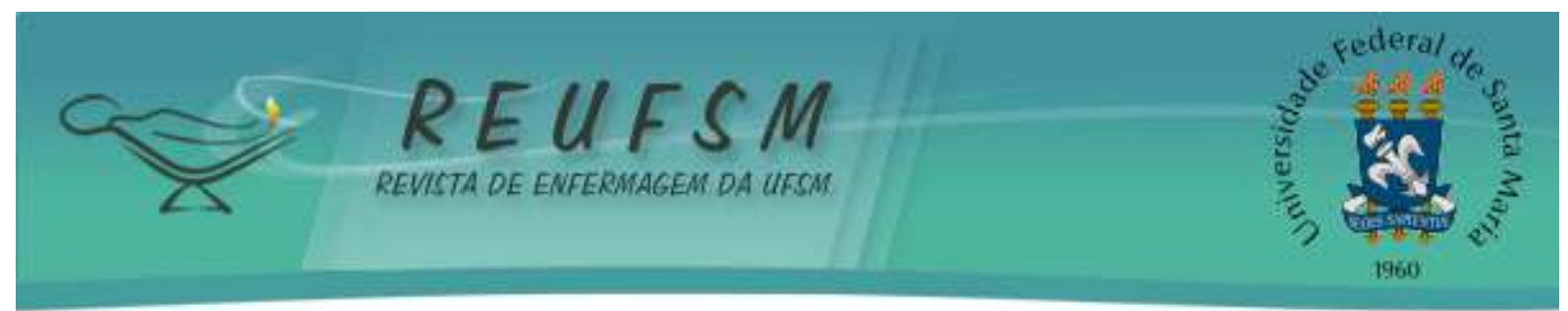

Verificou-se que a taxa de transmissão vertical do HIV, neste estudo, foi de $4,88 \%$, podendo ser associado à baixa adesão de consultas de pré-natal e, por conseguinte, a interrupção do ARV profilático durante a gestação, além do uso de drogas lícitas e ilícitas pelas gestantes com reflexos na saúde dos seus filhos.

A meta do plano municipal da saúde do município de Canoas contempla a erradicação da transmissão vertical do HIV exigindo ações que dependem, em parte, da atuação da equipe multidisciplinar, entre as quais do enfermeiro na orientação e encaminhamento da gestante ao longo da gestação. Cabe aos enfermeiros do município o envolvimento ativo na execução dessas ações, beneficiando as gestantes no pré-natal e, por conseguinte, os indicadores de saúde. As informações advindas deste estudo permitirão aos gestores do município planejar intervenções que possam contribuir na erradicação da transmissão perinatal do HIV como forma de contágio.

\section{REFERÊNCIAS}

1. Hofmann IC, Santos WM, Silva CB, Paula CC, Padoin SMM, Barros SMO. Fatores que interferem na transmissão vertical do HIV: revisão integrativa. Enferm Obstétrica. 2014;1(1):31-9.

2. Santos EM, Reis AC, Westman S, Alves RG. Avaliação do grau de implementação do programa de controle da transmissão vertical do HIV em maternidades do "Projeto Nascer". Escola Nacional de Saúde Pública Sérgio Arouca. Epidemiol Serv Saúde. 2010;19(3):257-69.

3. Friedrich L, Menegotto M, Magdaleno AM, Silva CLO. Transmissão vertical do HIV: uma revisão sobre o tema. Bol Cient Pediatr. 2016;05(3):81-6.

4. Kupek E, Oliveira JF. Transmissão vertical do HIV, da sífilis e da hepatite B no município de maior incidência de AIDS no Brasil: um estudo populacional no período de 2002 a 2007.

Rev Bras Epidemiol [Internet]. 2012 [acesso em 2014 nov 20];15(3):478-87. Disponível em: http://www.scielosp.org/pdf/rbepid/v15n3/04.pdf.

5. Ministério da Saúde. Secretaria de Vigilância em Saúde. Departamento de DST, Aids e Hepatites Virais. Protocolo clínico e diretrizes terapêuticas para manejo da infecção pelo HIV em crianças e adolescentes. 2014 [acesso em 2017 maio 05]. Disponível em:

http://conitec.gov.br/images/Protocolos/PCDT_Manejo-HIV-

CriancaseAdolescentes_2014.pdf.

6. Brasil. Ministério da Saúde. Boletim epidemiológico HIV- Aids e DST Ano IV - nº 1 - da $27^{\mathrm{a}}$ à $53^{\mathrm{a}}$ semana epidemiológica - julho a dezembro de 2014 Ano IV - $\mathrm{n}^{\mathrm{o}} 1$ - da $01^{\mathrm{a}}$ à $26^{\mathrm{a}}$ semana epidemiológica - janeiro a junho de 2015. Boletim de Aids e DST, Brasília (DF); 2015.

7. Instituto Brasileiro de Geografia e Estatística (IBGE). stimativas populacionais para os municípios e para as Unidades da Federação Brasil [Internet]. 2015 [acesso em 2016 jul 01]. Disponível em: www.ibge.gov.br/home/estatistica/populacao/estimativa2015/. 


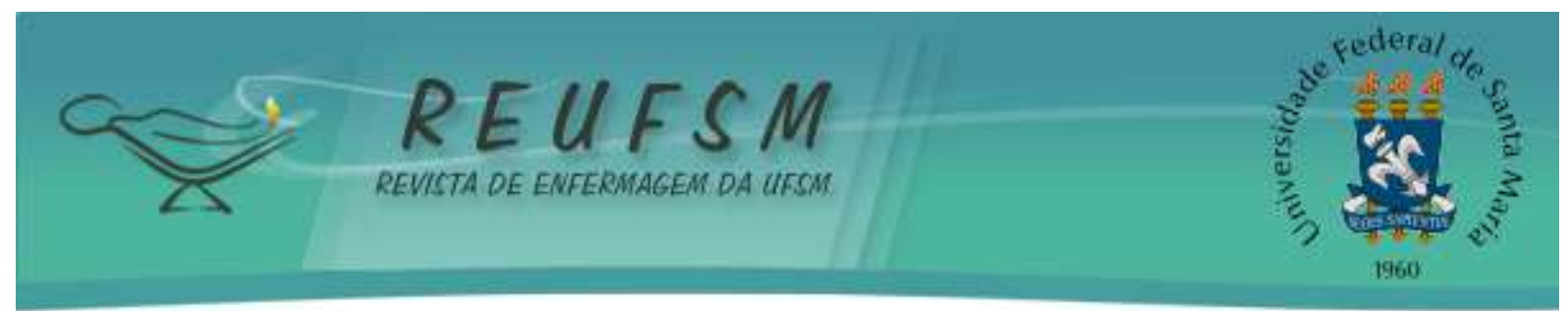

8. Lana FCF, Lima AS. Avaliação da prevenção da transmissão vertical do HIV em Belo Horizonte, MG (BR). Rev Bras Enferm. 2010;63(4):587-94.

9. Konopka CK, Beck ST, Wiggers D, Silva AK; Dieh FP, Santos FG. Perfil clínico e epidemiológico de gestantes infectadas pelo HIV em um serviço do sul do Brasil. Rev Bras Ginec Obstet. 2010;32(4):184-90.

10. Vieira ACBC, Miranda AE, Vargas PRM, Maciel ELN. Prevalência de HIV em gestantes e transmissão vertical segundo perfil socioeconômico, Vitória, ES (BR). Rev Saúde Pública. 2011;45(4):644-51.

11. Rodrigues STC, Vaz MJR, Barros SMO. Transmissão vertical do HIV em população atendida no serviço de referência. Acta Paul Enferm. 2013;26(2):158-64.

12. Teixeira M, Mendes MT, Borba KP, Pereira EM, Boba E. Avaliação da qualidade de vida de mulheres vivendo com HIV. Rev Enferm UFSM [Internet]. 2015 [acesso em 2015 ago 15]; 5(2):360-7. Disponivel em: https://periodicos.ufsm.br/reufsm/article/view/15277/pdf.

13. Figueiredo MRB, Thomé T, Pinto PC, Prates CS. Vivências de mães soropositivas para o HIV acompanhadas no Serviço de Assistência Especializada. Rev Enferm UFSM [Internet]. 2015 [acesso em 2015 ago 20];5(4):638-49. Disponível em:

https://periodicos.ufsm.br/reufsm/article/download/15406/pdf.

14. Brasil. Ministério da Saúde. Secretaria de Políticas de Saúde. Departamento de Ações Programáticas Estratégicas. Gestação de alto risco - manual técnico. $5^{\circ}$ ed. Brasília (DF): Ministério da Saúde; 2012.

15. Prefeitura de Canoas, RS. Secretaria Municipal de Saúde. Plano municipal de saúde 2014-2017. Canoas, 2013 [acesso em 2016 maio 13]. Disponível em: http://www.canoas.rs.gov.br/uploads/paginadinamica/335471/PMS_CANOAS_2014_2017.pdf.

16. Lima CT, Oliveira DR, Rocha EG, Pereira MLD. Manejo clínico da gestante com HIV positivo nas maternidades de referência da região do Cariri. Esc Anna Nery. 2010;14(3):468-76.

17. Rigoni EF, Carvalho FT, Lopes RL, Piccinini CA, Gonçalves TR, Santos BR. Gestação e HIV: preditores da adesão ao tratamento no contexto do pré-natal. Psic: Teor e Pesq. 2014;30(2):197-203.

Data de submissão: 21/08/2016

Data de aceite: $17 / 08 / 2017$

Contato do autor responsável: Maria Renita Burg Figueiredo

Endereço: Av. Farroupilha 8001 Bairro São José, Canoas. Cep: 92425-900

E-mail: renitaburgfigueredo@gmail.com 